\title{
JÓNSSON GROUPS OF VARIOUS CARDINALITIES
}

\author{
SAMUEL M. CORSON
}

\begin{abstract}
A group $G$ is Jónsson if $|H|<|G|$ whenever $H$ is a proper subgroup of $G$. Using an embedding theorem of Obraztsov it is shown that there exists a Jónsson group $G$ of infinite cardinality $\kappa$ if and only if there exists a Jónsson algebra of cardinality $\kappa$. Thus the question as to which cardinals admit a Jónsson group is wholly reduced to the well-studied question of which cardinals are not Jónsson. As a consequence there exist Jónsson groups of arbitrarily large cardinality. Another consequence is that the infinitary edge-orbit conjecture of Babai is true.
\end{abstract}

\section{INTRODUCTION}

A group $G$ is Jónsson (named for Bjarni Jónsson) if every proper subgroup of $G$ has cardinality strictly less than that of $G$. Finite groups are Jónsson and countably infinite examples include the quasi-cyclic groups $\mathbb{Z}\left(p^{\infty}\right)$ and the Tarski monsters constructed by Ol'shanskii ([8], [9, Theorem 28.1]). A Jónsson group of cardinality $\aleph_{1}$ was constructed by Shelah in [10] and further examples of Jónsson groups of cardinality $\aleph_{1}$ with striking properties were later obtained by Obraztsov (6], 9 , Corollary 35.4]).

There are known to exist Jónsson groups of cardinality $\aleph_{2}, \aleph_{3}, \ldots$ (see [5, Theorem E]) but there do not appear to be any of cardinality $\aleph_{\omega}$ or higher in the literature which are constructed only from the standard ZFC axioms. It is known that if there is a Jónsson group of cardinality $\kappa$ then there exists one of cardinaity $\kappa^{+}$[5], where $\kappa^{+}$denotes the successor cardinal to $\kappa$ (the smallest cardinal which is strictly greater than $\kappa$ ). If $2^{\lambda}=\lambda^{+}$then there exists a Jónsson group of cardinality $\lambda^{+}$[10, but the failure of this equality at every cardinal $\lambda>1$ is consistent with ZFC, modulo some large cardinal assumptions [3].

Before stating the main theorem we recall some definitions. Recall that an algebra is an ordered pair $(A, \mathcal{F})$ where $A$ is a set and $\mathcal{F}$ is a collection of finitary operations on $A$. An algebra $(A, \mathcal{F})$ is Jónsson if $\mathcal{F}$ is countable and any proper subalgebra is of cardinality strictly less than $|A|$. The main result is the following.

Theorem 1. For $\kappa$ an uncountable cardinal, there exists a Jónsson algebra of cardinality $\kappa$ if and only if there exists a simple, torsion-free Jónsson group of cardinality $\kappa$.

Naturally a Jónsson group is a Jónsson algebra, so we are only concerned with producing a Jónsson group given the existence of a Jónsson algebra. From Theorem 1 one obtains many new examples of Jónsson groups.

2010 Mathematics Subject Classification. 20A15, $20 \mathrm{E} 15$.

Key words and phrases. Jónsson group, Jónsson algebra.

This work was supported by the Heilbronn Institute for Mathematical Research, Bristol, UK.. 
Corollary 1. There is a Jónsson group of cardinality $\kappa$ when

(1) $\kappa$ is the successor of a regular cardinal [14;

(2) $\kappa=\lambda^{+}$, where $\lambda$ is singular and not a limit of weakly inaccessible cardinals 11]; or

(3) $\kappa=\beth_{\omega}^{+}[12$.

For example, there exist Jónsson groups of cardinality $\aleph_{1}, \aleph_{2}, \aleph_{\omega+1}, \aleph_{\omega^{2}+7}$, and $\aleph_{\omega_{1}+1}$. Since the successor of a cardinal is regular, we obtain from (1) that for each infinite cardinal $\kappa$ there exists a Jónsson group of cardinality $\kappa^{++}$. Thus there are Jónsson groups of arbitrarily large (regular) cardinality and this in itself has a consequence in combinatorics which we mention.

The infinitary edge-orbit conjecture of L. Babai [1, Conjecture 5.22] is the following:

For each cardinal $\kappa$ there exists a group $G$ such that whenever $G \simeq \operatorname{Aut}(\Gamma)$ for some graph $\Gamma$ the action of Aut $(\Gamma)$ on the edges of $\Gamma$ has at least $\kappa$ orbits.

It was shown by Simon Thomas that this conjecture is true provided there exist Jónsson groups of arbitrarily large regular cardinality [13] and so the following is immediate.

Corollary 2. The infinitary edge-orbit conjecture is true.

For another application, one can combine Theorem 1 with [5, Theorem G] to see that for any infinite cardinal $\kappa$ there exists a topological group $H$ of cardinality $\kappa^{+++}$ which is not discrete, with $H$ simple and Jónsson and every proper subgroup of $H$ is discrete. Keisler and Rowbottom have shown that there are Jónsson algebras of every infinite cardinality in Gödel's constructible universe $L$ [2, Corollary 9.1] and so in the constructible universe there exist Jónsson groups of every nonzero cardinality.

We caution the reader that there are some results about Jónsson algebras which do not carry over into the area of Jónsson groups. Recall that an algebra is locally finite if each finitely generated subalgebra is finite. It is known that there exists a Jónsson algebra of cardinality $\kappa$ if and only if there exists a locally finite Jónsson algebra of cardinality $\kappa[2$, Theorem 3.10]. On the other hand if $\kappa$ is an uncountable regular cardinal then there cannot exist a locally finite Jónsson group of cardinality $\kappa$ [4, Theorem 2.6].

\section{THE PROOF}

The proof of Theorem 11 will make use of a classical result regarding Jónsson algebras as well as a group embedding theorem. The following is obtained from a result of Łos (see [2, Theorem 3.4]).

Lemma 2. There is a Jónsson algebra on an infinite set $X$ if and only if there exists a binary operation $j: X \times X \rightarrow X$ such that $(X,\{j\})$ is Jónsson.

We will prepare to state a group embedding theorem by recalling some definitions. For $X$ a set we let $\mathcal{P}(X)$ denote the powerset of $X$ and $\mathcal{P}_{\text {fin }}(X)$ denote the collection of finite subsets of $X$.

Definitions 3. Given a collection of nontrivial groups without involutions $\left\{G_{i}\right\}_{i \in I}$ we call the set $\cup_{i \in I} G_{i}$, where $\{1\}=G_{i} \cap G_{j}$ whenever $i \neq j$, the free amalgam of the groups $\left\{G_{i}\right\}_{i \in I}$ and will denote it by $\Omega^{1}$. We will let $\Omega \subseteq \Omega^{1}$ denote the subset 
$\Omega^{1} \backslash\{1\}$. An embedding of $\Omega^{1}$ to a group $G$ is an injective function $E: \Omega^{1} \rightarrow G$ where each restriction $E \uparrow G_{i}$ is a homomorphism. An embedding $E$ extends to a homomorphism $\phi$ from the free product $\star_{i \in I} G_{i}$ to $G$, and since each $\phi \uparrow G_{i}$ is an isomorphism, we consider each $G_{i}$ to be a subgroup of $G$ in such a situation.

Definition 4. Let $\left\{G_{i}\right\}_{i \in I}$ be a collection of nontrivial groups without involutions, $\Omega^{1}$ be the free amalgam, and $\Omega=\Omega^{1} \backslash\{1\}$ as above. A function $f: \mathcal{P}(\Omega) \backslash\{\varnothing\} \rightarrow$ $\mathcal{P}(\Omega)$ is generating provided

(a) if $X \subseteq G_{i}$ for some $i \in I$ then $f(X)=\langle X\rangle \backslash\{1\}$;

(b) if $X \subseteq \Omega$ is finite and $X \nsubseteq G_{i}$ for all $i \in I$ then $f(X)=Y$ where $Y$ is a countable subset of $\Omega$ such that $X \subseteq Y$ and if $Z \subseteq Y$ is finite nonempty then $f(Z) \subseteq Y$;

(c) if $X \subseteq \Omega$ is infinite and $X \nsubseteq G_{i}$ for all $i \in I$ then $f(X)=\cup_{Z \in \mathcal{P}_{\text {fin }}(X) \backslash\{\varnothing\}} f(Z)$.

The following is a special case of a beautiful embedding theorem of Obraztsov [7. Theorem A].

Proposition 5. Suppose that $\left\{G_{i}\right\}_{i \in I}$ is a collection of nontrivial groups without involutions and that $f$ is a generating function on this collection, with $|I| \geq 2$. Then there is a simple group $G$ and an embedding $E: \Omega_{1} \rightarrow G$ of the free amalgam which induces a homomorphism $\phi: *_{i \in I} G_{i} \rightarrow G$ satisfying the following properties:

(1) $\phi$ is surjective;

(2) if $h \in G$ is not conjugate in $G$ to an element in one of the groups $G_{i}$ then $h$ is of infinite order;

(3) each subgroup $M$ of $G$ is either cyclic, or conjugate in $G$ to a subgroup of one of the $G_{i}$, or conjugate in $G$ to a subgroup of form $\langle C\rangle$ where $C=f(X)$ for some $\varnothing \neq X \subseteq \Omega$.

Proof of Theorem 11. Suppose that there is a Jónsson algebra of uncountable cardinality $\kappa$. Let $\left\{G_{\alpha}\right\}_{\alpha<\kappa}$ be a collection of groups where each $G_{\alpha}$ is infinite cyclic and generated by $z_{\alpha}$. Let $\Omega^{1}$ be the free amalgam of the groups $\left\{G_{\alpha}\right\}_{\alpha<\kappa}$ and $\Omega=\Omega^{1} \backslash\{1\}$. Let $\tau: \Omega \rightarrow\left\{z_{\alpha}\right\}_{\alpha<\kappa}$ be given by $g \mapsto z_{\alpha}$ where $g \in G_{\alpha}$.

As there is a Jónsson algebra of cardinality $\kappa$, we have by Lemma 2 a binary operation $j:\left\{z_{\alpha}\right\}_{\alpha<\kappa} \times\left\{z_{\alpha}\right\}_{\alpha<\kappa} \rightarrow\left\{z_{\alpha}\right\}_{\alpha<\kappa}$ such that $\left(\left\{z_{\alpha}\right\}_{\alpha<\kappa},\{j\}\right)$ is a Jónsson algebra. For each subset $Y \subseteq\left\{z_{\alpha}\right\}_{\alpha<\kappa}$ we let $\bar{j}(Y) \subseteq\left\{z_{\alpha}\right\}_{\alpha<\kappa}$ denote the subalgebra generated by $Y$ under the operation $j$. Let $\pi:\left\{z_{\alpha}\right\}_{\alpha<\kappa} \rightarrow \kappa$ be given by $z_{\alpha} \mapsto \alpha$.

We claim that the function $f: \mathcal{P}(\Omega) \backslash\{\varnothing\} \rightarrow \mathcal{P}(\Omega)$ defined by

$$
f(X)= \begin{cases}\langle X\rangle \backslash\{1\} & \text { if } X \subseteq G_{\alpha} \text { for some } \alpha \in \kappa \\ \left(\bigcup_{\alpha \in \pi(\bar{j}(\tau(X)))} G_{\alpha}\right) \backslash\{1\} & \text { otherwise }\end{cases}
$$

is generating. Certainly condition (a) of Definition 4 holds. To check condition (b) we let $X \subseteq \Omega$ be finite with $X \nsubseteq G_{\alpha}$ for all $\alpha<\kappa$. The set $\tau(X) \subseteq\left\{z_{\alpha}\right\}_{\alpha<\kappa}$ is finite, and so the set $\bar{j}(\tau(X))$ generated by $\tau(X)$ under $j$ will be countable. Then $\pi(\bar{j}(\tau(X)))$ is countable. Thus the set $f(X)=\left(\cup_{\alpha \in \pi(\bar{j}(\tau(X)))} G_{\alpha}\right) \backslash\{1\}$ is countable as a countable union of countable sets. Certainly $f(X) \supseteq X$ in this case, since

$$
X \subseteq\left(\bigcup_{\alpha \in \pi(\tau(X))} G_{\alpha}\right) \backslash\{1\} \subseteq\left(\bigcup_{\alpha \in \pi(\bar{j}(\tau(X)))} G_{\alpha}\right) \backslash\{1\}=f(X) .
$$

Moreover given a finite nonempty $Z \subseteq f(X)$ we either have $Z \subseteq G_{\alpha}$ for some $\alpha<\kappa$, in which case $\alpha \in \pi(\bar{j}(\tau(X)))$ and

$$
f(Z)=\langle Z\rangle \backslash\{1\} \subseteq G_{\alpha} \backslash\{1\} \subseteq f(X)
$$


or else we have

$$
f(Z)=\left(\bigcup_{\alpha \in \pi(\bar{j}(\tau(Z)))} G_{\alpha}\right) \backslash\{1\} \subseteq\left(\bigcup_{\alpha \in \pi(\bar{j}(\tau(X)))} G_{\alpha}\right) \backslash\{1\}=f(X)
$$

since $\tau(Z) \subseteq \bar{j}(\tau(X))$. Thus condition (b) holds. For condition (c) we let $X \subseteq \Omega$ be infinite such that $X \nsubseteq G_{\alpha}$ for each $\alpha \in \kappa$. Given $g \in f(X)$ we have $g \in G_{\alpha} \backslash\{1\}$ for some $\alpha \in \pi(\bar{j}(\tau(X)))$. Thus $z_{\alpha} \in \bar{j}(\tau(X))$ and we may select $Z_{0} \subseteq \tau(X)$ which is finite such that $z_{\alpha} \in \bar{j}\left(Z_{0}\right)$, and we may assume without loss of generality that $Z_{0}$ has at least two elements (since $\tau(X)$ has at least two elements). Select a finite $Z \subseteq X$ such that $\tau(Z)=Z_{0}$ and it is clear that $g \in f(Z)$. Therefore $f(X) \subseteq$ $\bigcup_{Z \in \mathcal{P}_{\text {fin }}(X) \backslash\{\varnothing\}} f(Z)$. To see the inclusion $f(X) \supseteq \bigcup_{Z \in \mathcal{P}_{\text {fin }}(X) \backslash\{\varnothing\}} f(Z)$ it is sufficient to show $f(X) \supseteq f(Z)$ for every $Z \in \mathcal{P}_{\text {fin }}(X) \backslash\{\varnothing\}$. This is almost the same check as in part (b): either $Z \subseteq G_{\alpha}$ for some $\alpha<\kappa$, so that $\alpha \in \pi(\tau(X)) \subseteq \pi(\bar{j}(\tau(X)))$ and

$$
f(Z)=\langle Z\rangle \backslash\{1\} \subseteq G_{\alpha} \backslash\{1\} \subseteq f(X)
$$

or else

$$
f(Z)=\left(\bigcup_{\alpha \in \pi(\bar{j}(\tau(Z)))} G_{\alpha}\right) \backslash\{1\} \subseteq\left(\bigcup_{\alpha \in \pi(\bar{j}(\tau(X)))} G_{\alpha}\right) \backslash\{1\}=f(X) .
$$

Thus $f(X)=\bigcup_{Z \in \mathcal{P}_{\text {fin }}(X) \backslash\{\varnothing\}} f(Z)$ and (c) holds.

We may therefore apply Proposition 5 to produce a simple group $G$ into which the free amalgam $\Omega^{1}$ embeds and satisfying the listed properties of Proposition 5 . Notice that $G$ is torsion-free by part (2) of Proposition 5, since each of the subgroups $G_{\alpha}$ is torsion-free. Also, $|G|=\kappa$ since $\Omega^{1}$ injects into $G$ and $G=\left\langle\bigcup_{\alpha<\kappa} G_{\alpha}\right\rangle$ with $\left|G_{\alpha}\right|=\aleph_{0}$ for each $\alpha<\kappa$.

It remains to see that $G$ is Jónsson. Letting $M$ be a subgroup of $G$ with $|M|=\kappa$, we know by part (3) of Proposition [5] that up to conjugation $M$ is equal to $\langle C\rangle$ where $C=f(X)$ for a nonempty $X \subseteq \Omega$. Thus by conjugating if necessary we may assume without loss of generality that $M=\langle C\rangle$ for such a $C$. Since $|M|=\kappa$ it is clear that $|C|=\kappa$ since $\kappa$ is uncountable. If $X$ were to satisfy $X \subseteq G_{\alpha}$ for some $\alpha<\kappa$ then $f(X)=C \subseteq G_{\alpha}$, but $C$ is uncountable, a contradiction. Thus $X$ is not a subset of any $G_{\alpha}$, and $X$ cannot be finite for then $C$ would be countable by condition (b). Therefore $C=f(X)=\cup_{Z \in \mathcal{P}_{\text {fin }}(X) \backslash\{\varnothing\}} f(Z)$. Since $f(Z)$ is countable for any $Z \in \mathcal{P}_{\text {fin }}(X) \backslash\{\varnothing\}$, and $\left|\mathcal{P}_{\text {fin }}(X) \backslash\{\varnothing\}\right|=|X|$ since $X$ is infinite, we see that $\kappa=|C| \leq|X| \cdot \aleph_{0}$, and therefore $|X|=\kappa$.

Since the function $\tau: \Omega \rightarrow\left\{z_{\alpha}\right\}_{\alpha<\kappa}$ is countable-to-one we see that $|\tau(X)|=\kappa$, and as $j$ is as in Lemma 2 we get $\bar{j}(\tau(X))=\left\{z_{\alpha}\right\}_{\alpha<\kappa}$. Thus $\pi(\bar{j}(\tau(X)))=\kappa$ and

$$
C=f(X)=\left(\cup_{\alpha \in \pi(\bar{j}(\tau(X)))} G_{\alpha}\right) \backslash\{1\}=\Omega
$$

from which we have $M=\langle\Omega\rangle=G$ and we are done.

\section{ACKNOWLEDGEMENT}

The author gives thanks to the anonymous referee for careful reading and suggested improvements of the paper. 


\section{REFERENCES}

[1] L. Babai, On the abstract group of automorphisms, in Combinatorics (Temperley, editor), London Mathematical Society Lecture Note Series 52 (1981), 1-40.

[2] K. J. Devlin, Some weak versions of large cardinal axioms, Ann. Math. Log. 5 (1973), 291-325.

[3] M. Foreman, W. H. Woodin, The generalized continuum hypothesis can fail everywhere, Ann. Math. 133 (1991), 1-35.

[4] O. H. Kegel, B. A. F. Wehrfritz, Locally Finite Groups, North-Holland, Amsterdam (1973).

[5] S. Morris, V. N. Obraztsov, Nondiscrete topological groups with many discrete subgroups, Topology Appl. 84 (1998), 105-120.

[6] V. N. Obraztsov, An embedding theorem for groups and its corollaries, Mat. Sb. 180 (1989), 529-541.

[7] V. N. Obraztsov, A new embedding scheme for groups and some applications, J. Austral. Math. Soc. (Series A) 61 (1996), 267-288.

[8] A. Yu. Ol'shanskii, An infinite group with subgroups of prime orders, Izv. Akad. Nauk SSSR, Ser. Mat. 44 (1980), 309-321.

[9] A. Yu. Ol'shanskii, Geometry of Defining Relations in Groups, Kluwer Academic Publisher (1991).

[10] S. Shelah, On a problem of Kurosh, Jónsson groups, and applications, Word Problems II. North-Holland Publ. Company (1980), 373-394.

[11] S. Shelah, Was Sierpiński right? I, Israel J. Math. 62 (1988), 355-380.

[12] S. Shelah, More Jonsson algebras, Arch. Math. Log. 42 (2003), 1-44.

[13] S. Thomas, The infinite case of the edge-orbit conjecture, Algebra Univers. 24 (1987), 167168.

[14] J. Tryba, On Jónsson cardinals with uncountable cofinality, Israel J. Math 49 (1984), 315-324.

School of Mathematics, University of Bristol, Fry Building, Woodland Road, BrisTOL, BS8 1UG, United Kingdom.

Email address: sammyc973@gmail.com 